\title{
1) Lumbar Artery Hemorhage Complicating Surgical Spine Intervention: Treatment by Transcatheter Embolization
}

Vascular injuries complicating lumbar spine surgery are uncommon and mostly involve the great vessels, either arteries or veins, situated anterior to the spine. We report a case of an iatrogenic lumbar artery injury resulting in massive retroperitoneal hemorrhage. Surgical hemostasis was insufficient, but the bleeding could be completely stopped by transcatheter embolization. To our knowledge, iatrogenic injury of a lumbar artery during surgical intervention successfully managed by transcatheter embolization is not yet reported in the literature till now.

\section{Case Report}

A 56-year-old woman underwent a left total mastectomy for invasive mamma carcinoma 8 years ago. Two years later she developed a rib metastasis and 4 years later a thoracic (D12) and lumbar (L5) vertebral bone metastasis. These 
metastases were treated palliatively by radio- and chemotherapy. Six years after mastectomy an epidural metastasis at the level D9-D10 and progression of the D12-metastasis were found. Because of progressive symptoms of pain it was decided to perform palliative surgery by means of interlaminary fenestration at the level D9-D10 and dorsal spondylosyndesis at the level D9-L2.

One month ago loosening of the metallic spondylosyndesis material was detected implicating the risk of vertebral collapse and paraparesis. It was decided to perform surgical reintervention by placement of some new spondylosyndesis material at the level D3-L2. Removal of the left distal pedicular screw which was surrounded by a large amount of tumoral tissue resulted in a severe bleeding which could be stopped by manual compression. After removal of the loosened spondylosyndesis material and placement of new screws and spondylosyndesis material, all layers were quickly sutured to provoke compartmentalization and cessation of the bleeding although the bleeding vessel could not be identified macroscopically. A few hours after the operation, the patient became hemodynamically unstable and after infusion of 4 units of blood ( $500 \mathrm{ml} / \mathrm{unit}$ ), she was immediately referred to the angiography suite. Flush aortography (Fig. 1) from a right femoral approach, revealed massive left paraaortic extravasation of contrast medium. Selective catheterization of the ostium of the left second lumbar artery (Fig. 2) (Cobra C-2 glidecath, Terumo, Leuven, Belgium) confirmed the extravasation into an already huge retroperitoneal hematoma. Superselective catheterization by coaxial microcatheter (SPmicrofocus, Terumo, Leuven, Belgium) made emboliza-tion by fibered platinum microcoils (Target Therapeutics, Fremont, CA, USA) effective and without danger to drop a coil into the aorta. Because of the life-threatening situation, there was no time or need to identify the arteria radicularis magna before (or after) the embolization. Control angiography after embolization (Fig. 3 and $\mathbf{4}$ ) showed complete postostial occlusion of the second left lumbar artery. The patient became hemodynamically stable and could be extubated 5 days later. Three weeks after the intervention the patient left the hospital and receives a palliative home-treatment.

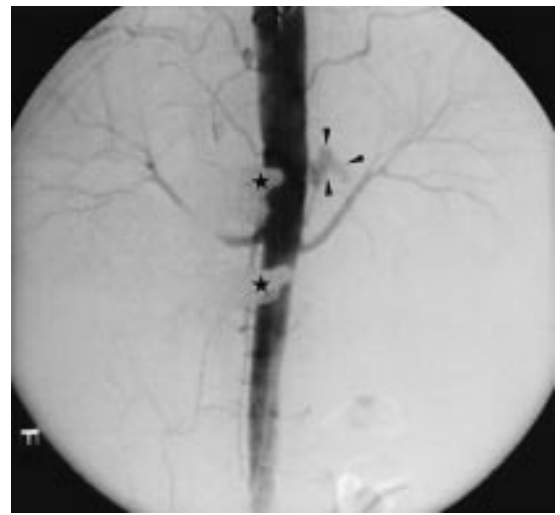

Fig. 1 Flush aortography shows massive, left paraaortic contrast extravasation (arrowheads). Not also the osteosynthesis material projecting in the right paraaortic region (asterisk).

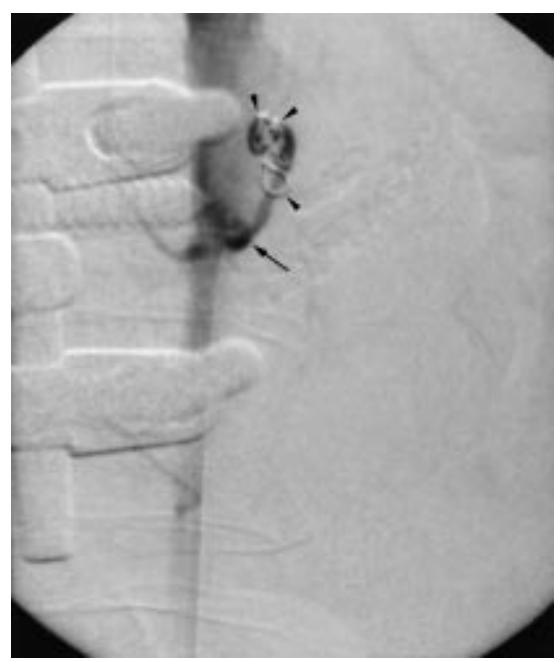

Fig. 3 Selective control angiography after embolization shows the presence of coils (arrowheads) located in the postostial segment of the second left lumbar artery (arrow), absence of any contrast extravasation; reflux of contrast medium into the aorta.

\section{Discussion}

Vascular injuries following surgical spine intervention are rare and mostly occur after disectomy. Because of the anatomical localization anterior to the lumbar spine, the aorta, caval vein and iliac vessels are mostly involved by direct tears, pseudoaneurysms and arteriovenous fistulas. Rarely other vessels can be damaged during spine surgery like an iliolumbar vein, a lumbar artery, or an accessory inferior polar artery of a fused kidney.

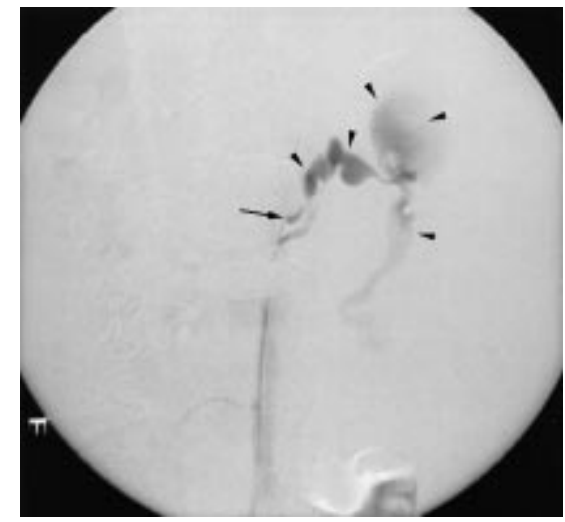

Fig. 2 Selective injection of the second left lumbar artery confirms the massive contrast extravasation (arrowheads).

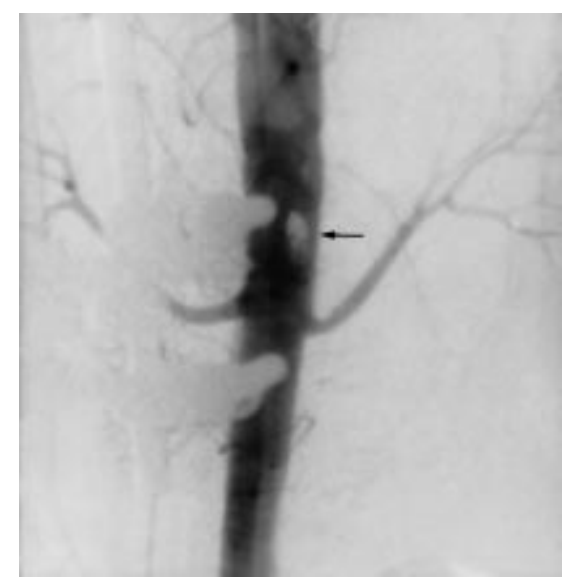

Fig. 4 Flush aortography after embolization shows absence of any contrast extravasation and the projection of coils (arrow) in the aorta.

Retroperitoneal hemorrhage due to a lumbar artery injury can be provoked by iatrogenic (Kim et al., Radiology 1987; 165: 175) and non-iatrogenic trauma (Scalafani et al., Cardiovasc Intervent Radiol 1991; 14: 175) or can occur "spontaneously" during anticoagulant therapy (Kalinowski and Trerotola, Cardiovasc Intervent Radiol 1998; 21: 337).

Angiography remains the golden standard to diagnose a lumbar artery lesion. Angiographic findings of bleeding include extravasation, as this case clearly demonstrates, pseudoaneurysm (Ikubo et al., Surgery Today Jpn J Surg 1993; 23: $635)$ and vertebral staining (Sclafani et al., Radiology 1987; 165: 709). 
Operative control of lumbar artery bleeding is often difficult, since the site of origin may not be readily isolated, especially in the situation of retroperitoneal hematoma formation. In these situations, transcatheter embolization appears to be successful and minimally invasive. Embolic agents mostly used to occlude a lumbar artery are polyvinyl alcohol particles, coils and gel sponge. Although very efficient and minimally invasive, lumbar artery embolization is not always without complication. Spinal cord infarction, mostly due to the occlusion of the great anterior radicular artery (Adamkiewitz), is the most dramatic complication of lumbar artery occlusion, but also peripheral nerve infarction or paraspinal muscle infarction are described in the literature (Doppman and DiChiro, Radiology 1976; 119: 609).

- Massive lumbar artery hemorrhage complicating orthopedic spine surgery, is a very rare iatrogenic condition which can be difficult to diagnose and subsequently to manage efficiently perioperatively.

- Transcatheter embolization of the bleeding lumbar artery seems to be minimally invasive and a very efficient tool to stop the bleeding immediately and completely.

G. Maleux, J. Samson, L. Stockx, Leuven, Belgium 